\title{
Short-Term Immunogenicity Profiles and Predictors for Suboptimal Immune Responses in Patients with End-Stage Kidney Disease Immunized with Inactivated SARS-CoV-2 Vaccine
}

\author{
Sarinya Boongird · Piyatida Chuengsaman · Chavachol Setthaudom • \\ Arkom Nongnuch · Montira Assanatham · Salinnart Phanprasert • \\ Rungthiwa Kitpermkiat - Sasisopin Kiertiburanakul · Kumthorn Malathum • \\ Angsana Phuphuakrat · Andrew Davenport · Jackrapong Bruminhent
}

Received: October 29, 2021 / Accepted: November 23, 2021 / Published online: December 3, 2021

(C) The Author(s) 2021

\section{ABSTRACT}

Introduction: Patients with end-stage kidney disease (ESKD) are at risk of severe coronavirus disease and mortality. Immunogenicity of severe acute respiratory syndrome coronavirus 2 (SARS-CoV-2) inactivated whole-virus vaccine in patients with ESKD has never been explored.

S. Boongird · A. Nongnuch · M. Assanatham .

S. Phanprasert · R. Kitpermkiat

Division of Nephrology, Department of Medicine, Faculty of Medicine Ramathibodi Hospital, Mahidol University, Bangkok, Thailand

P. Chuengsaman

Banphaeo-Charoenkrung Peritoneal Dialysis Center, Banphaeo Dialysis Group, Banphaeo Hospital,

Bangkok, Thailand

C. Setthaudom

Immunology Laboratory, Department of Pathology, Faculty of Medicine Ramathibodi Hospital, Mahidol University, Bangkok, Thailand

S. Kiertiburanakul · K. Malathum - A. Phuphuakrat · J. Bruminhent $(\square)$

Division of Infectious Diseases, Department of Medicine, Faculty of Medicine Ramathibodi

Hospital, Mahidol University, 270 Rama VI Road, Ratchathewi, Bangkok 10400, Thailand

e-mail: jackrapong.brm@mahidol.ac.th

A. Davenport

UCL Centre for Nephrology, Royal Free Hospital, University College London, London, UK
Methods: We conducted a prospective cohort study of 60 patients with ESKD and 30 healthy controls. All participants received two doses of an inactivated whole-virus SARS-CoV-2 vaccine (Sinovac Biotech Ltd) 4 weeks apart. SARS-CoV2-specific humoral and cell-mediated immune responses were investigated and referenced with healthy controls.

Results: After two doses, an anti-receptorbinding domain immunoglobulin $\mathrm{G}$ of $50 \mathrm{AU} /$ $\mathrm{ml}$ or greater was present in 53 of 60 patients $(88 \%)$ in the ESKD group and all participants $(100 \%)$ in the control group $(P=0.05)$. The percentage of patients with ESKD and controls with neutralizing antibodies of 35\% threshold or greater was $58 \%$ and $88 \%$, respectively $(P=0.01)$. Furthermore, the proportion of patients with ESKD and S1-specific T cell response was comparable with controls ( $82 \%$ vs. $77 \%, P=0.45)$. Old age, high ferritin level, and low absolute lymphocyte count were independently associated with poor humoral immune responses.

Conclusions: Patients with ESKD could develop similar SARS-CoV-2-specific cell-mediated immune responses compared to healthy controls, although suboptimal humoral immune responses were observed following two doses of SARS-CoV-2 vaccination. Therefore, patients with ESKD and the abovementioned factors are at risk of generating inadequate humoral 
immune responses, and a vaccine strategy to elicit greater immunogenicity among these relatively immunocompromised patients is warranted. (Thai Clinical Trials Registry, TCTR20210226002).

Keywords: COVID-19; Dialysis; Inactivated vaccine; Neutralizing antibody; Receptorbinding domain

\section{Key Summary Points}

\section{Why carry out this study?}

End-stage kidney disease (ESKD) patients who are infected with severe acute respiratory syndrome coronavirus 2 (SARS-CoV-2) are at risk of high morbidity and mortality.

Immunogenicity profiles of an inactivated whole-virus SARS-CoV-2 vaccine (CoronaVac) in patients with ESKD receiving dialysis have not been investigated.

We aimed to evaluate SARS-CoV-2-specific humoral and cell-mediated immune responses following two doses of the inactivated whole-virus SARS-CoV-2 vaccine, with a 4 -week interval between doses, in patients with ESKD receiving dialysis and compared to healthy individuals.

\section{What was learned from the study?}

Patients with ESKD could develop similar SARS-CoV-2-specific cell-mediated immune responses, although suboptimal humoral immune responses were observed following the standard two doses of the inactivated SARS-CoV-2 vaccine.

Patients with ESKD who have old age, high ferritin level, and low absolute lymphocyte count are at increased risk of inadequate humoral immune responses.
Patients with ESKD may benefit from routine monitoring of vaccine-generated immune responses, and a vaccine strategy to elicit greater immunogenicity among these relatively immunocompromised patients is warranted.

\section{INTRODUCTION}

Severe acute respiratory syndrome coronavirus 2 (SARS-CoV-2) is a highly contagious pathogenic virus causing an ongoing worldwide pandemic of coronavirus disease 2019 (COVID19). Patients with end-stage kidney disease (ESKD) are susceptible to SARS-CoV-2 infection and serious complications $[1,2]$. Thus, providing a safe and effective SARS-CoV-2 vaccine to patients with ESKD should be immediately implemented to reduce unfavorable outcomes. Immunogenicity data of different types of SARSCoV-2 vaccines are rapidly evolving in the general population; however, the immunogenicity profiles of SARS-CoV-2 vaccines among patients with ESKD remain limited [3].

Preliminary analyses have suggested that SARS-CoV-2 messenger RNA (mRNA) vaccines mount high immunogenicity among patients with ESKD [4-9]. Since these mRNA vaccines are only available in some countries, different forms of SARS-CoV-2 vaccines such as viral vector-based and inactivated vaccines have been administered to patients with ESKD with insufficient data supporting their efficacies or immunogenicity in this population. An inactivated whole-virus SARS-CoV-2 vaccine (CoronaVac) is one of the most widely used vaccines worldwide. However, data on immunogenicity profiles of an inactivated SARS-CoV-2 vaccine (CoronaVac) in patients undergoing dialysis are limited. Our preliminary report revealed that patients with ESKD receiving dialysis generated a weak antibody response following two doses of the inactivated SARS-CoV-2 vaccine compared to healthy individuals [10]. Moreover, there are concerns that patients with ESKD may not adequately develop protective immunity following immunization with an inactivated 
SARS-CoV-2 vaccine, as previously observed with the hepatitis $B$ and influenza vaccinations [11-13]. Consequently, the question arises of whether inactivated whole-virus SARS-CoV-2 vaccines are adequately immunogenic in patients with ESKD, many of whom are considered relatively immunocompromised. We therefore evaluated SARS-CoV-2-specific humoral and cell-mediated immune responses following two doses of the inactivated wholevirus SARS-CoV-2 vaccine, with a 4 -week interval between doses, among these patients.

\section{METHODS}

\section{Study Design and Participants}

We conducted a prospective cohort study between April 2021 and July 2021 at the Faculty of Medicine Ramathibodi Hospital, Mahidol University, Bangkok, Thailand. The study included two cohorts: patients with ESKD on dialysis (the ESKD group) and healthy controls (the control group). Patients with ESKD were screened and recruited from two hospitals: Ramathibodi Hospital and Banphaeo General Hospital (Charoenkrung branch), both located in Bangkok, Thailand.

The ESKD included adult patients aged between 18 and 59 years, who had been stable for more than 1 month on their dialysis prescriptions. Participants in the control group were volunteers from our community who had a similar age range to the ESKD group and normal kidney function. Participants were excluded if they had concurrent active infections, had received vaccinations (COVID-19 or others) within 1 month, had been diagnosed with COVID-19 before enrollment, or were experiencing respiratory tract symptoms.

All participants received two doses of the inactivated whole-virus SARS-CoV-2 vaccine (CoronaVac, Sinovac Biotech Ltd., China), containing $3 \mu \mathrm{g}$ of inactivated SARS-CoV-2 administered intramuscularly, 4 weeks apart.

\section{Immunogenicity Outcomes}

SARS-CoV-2-specific humoral and cell-mediated immune responses of an inactivated wholevirus SARS-CoV-2 vaccine were evaluated at baseline, 4 weeks after the first dose, and 2 weeks after the second dose. Our initial study reported the levels of immunoglobulin G (IgG) antibodies against the receptor-binding domain (RBD) of the SARS-CoV-2 spike protein (antiRBD IgG) using a SARS-CoV-2 immunoglobulin $\mathrm{G}$ assay [10]. In the present study, the neutralizing antibody response, which represents the functional effects of the anti-RBD IgG, was assessed using a SARS-CoV-2 surrogate virus neutralization test (sVNT). Cell-mediated immune response was assessed using an interferon- $\gamma$ enzyme-linked immunospot (IFN $\gamma$-ELISpot) assay.

\section{SARS-CoV-2-Specific Humoral Immune Response}

Plasma concentration of anti-RBD IgG was quantitated using a chemiluminescent microparticle immunoassay (Abbott SARS-CoV2 IgG II Quant assay; Abbott, Lake Bluff, IL, USA) and analyzed on the Abbott Architect instrument according to the manufacturer's instructions. Levels of anti-RBD IgG were reported in arbitrary units (AU)/ml. Seroconversion was defined as anti-RBD IgG titers $\geq 50 \mathrm{AU} / \mathrm{ml}$ [14]. This cutoff value provides a sensitivity of $91.6 \%$ (95\% confidence interval $[95 \% \mathrm{CI}]$ 85.8-95.6\%) and a specificity of $99.4 \%$ (95\% CI $97.1-100 \%)$ [14, 15].

The neutralizing antibodies (neuAbs) were quantitatively assessed using an sVNT (Euroimmun kits, Lübeck, Germany). This technique indirectly measures the percentage of neuAb inhibition in the serum by calculating the binding inhibition potency between the angiotensin-converting enzyme 2 receptor and the receptor-binding domain in samples and controls [16]. According to the manufacturer's instructions, this cutoff provides a diagnostic sensitivity of $95.9 \%$ and a specificity of $99.7 \%$ [17], and at least 35\% inhibition was classed as demonstrating neuAb seropositivity. 


\section{SARS-CoV-2-Specific Cell-Mediated Immune Response}

Assessments of SARS-CoV-2-specific T cell responses were determined using a method previously published by Zuo et al. [18]. In brief, we measured the levels of interferon-gamma (IFN $\gamma$ ) production by activated peripheral blood mononuclear cells (PBMCs) following stimulation with SARS-CoV-2 mixed peptide pools, using a human IFN $\gamma$ ELISpot plus ALP kit (Mabtech, Stockholm, Sweden). Isolated PBMCs were normalized to $2.5 \times 10^{6}$ cells $/ \mathrm{ml}$ in $100 \mu \mathrm{l}$ AIM-V medium (Gibco, Waltham, MA, USA) and stimulated under five different conditions: (1) AIM-V medium (negative control); (2) a scanning peptide pool covering the S1 domain (S1) of the SARS-CoV-2 spike protein (Mabtech); (3) a scanning peptide pool based on epitopes from S2 domain and nucleoprotein (S2N) (Mabtech); (4) SARS-CoV-2 spike protein, nucleoprotein, membrane protein, open reading frame (ORF)-3a, and ORF-7a (SNMO) defined peptide pool (Mabtech); and (5) antiCD3 antibody (positive control). The number and quality of reactive spots were analyzed using an ImmunoSpot analyzer (Cellular Technology Limited, Shaker Heights, OH, USA) and ImmunoSpot Software v5.0.9.15 (Cellular Technology Limited), respectively. Magnitudes of $\mathrm{T}$ cell responses were presented as medians with interquartile ranges (IQRs) of IFN $\gamma$-producing spot forming units (SFUs) per $10^{6}$ PBMCs. Participants who generated any detectable level of T cell response after stimulation with a scanning pool of SARS-CoV-2 S1 domain of the spike protein were classified as cell-mediated immune responders.

\section{Statistical Analyses}

Data were analyzed for normality before analysis. Descriptive statistics are displayed with numbers (percentage) for categorical variables and mean (standard deviation, SD) or median (IQR) for continuous variables, depending on the distribution of data. Baseline characteristics between patients receiving hemodialysis (HD) and peritoneal dialysis (PD) were compared using the Student $t$ test and the Fisher's exact test for continuous and categorical variables, respectively. The Mann-Whitney $U$ test was used to compare vaccine-elicited immunogenicity between patients undergoing HD and PD and between patients with ESKD and healthy controls. The Wilcoxon signed-rank test was used to compare median humoral and cell-mediated immune responses within groups at different time points. For all categorical variables, the chi-square test or Fisher's exact test was used for comparisons between two groups. Values of $P<0.05$ were considered statistically significant. Factors associated with being neuAb seronegative (percentage neuAb inhibition less than 35\%) after vaccination were identified using a stepwise logistic regression method. Any variables with a $P$ value $<0.1$ from univariate analysis were included in a multiple logistic regression model. Statistical analyses were carried out using Stata version 15 (StataCorp, LLC, College Station, TX, USA). All graphs were plotted using GraphPad Prism 6.0 (GraphPad Software, Inc, San Diego, CA, USA).

\section{Ethics Approval}

The Institutional Review Board of the Faculty of Medicine, Ramathibodi Hospital, Mahidol University, Bangkok, Thailand approved the study protocol (approval number MURA2021/ 242). Participation in this study was discussed with the patients from Banphaeo General Hospital (Charoenkrung branch). The patients agreed to the referral, including sharing information about their condition and the Institutional Review Board of Banphaeo General Hospital (Charoenkrung branch) approved the release of patients' information. All participants provided written informed consent before enrollment. The study was conducted following the principles laid out in the declaration of Helsinki and was registered with the Thai Clinical Trials Registry (TCTR20210226002). 


\section{RESULTS}

\section{Baseline Characteristics of Patients with ESKD and Healthy Controls}

Sixty patients with ESKD (31 patients undergoing $\mathrm{HD}$ and $29 \mathrm{PD}$ ) and 30 healthy controls were recruited, all of whom received two doses of the vaccine and completed all visits (Fig. 1). Baseline demographic data for the patients undergoing HD and PD are shown in Table 1. There were no differences in age, body mass index (BMI), gender, dialysis vintage, or prevalence of diabetes mellitus between the HD and PD groups. Only one patient in the PD group had

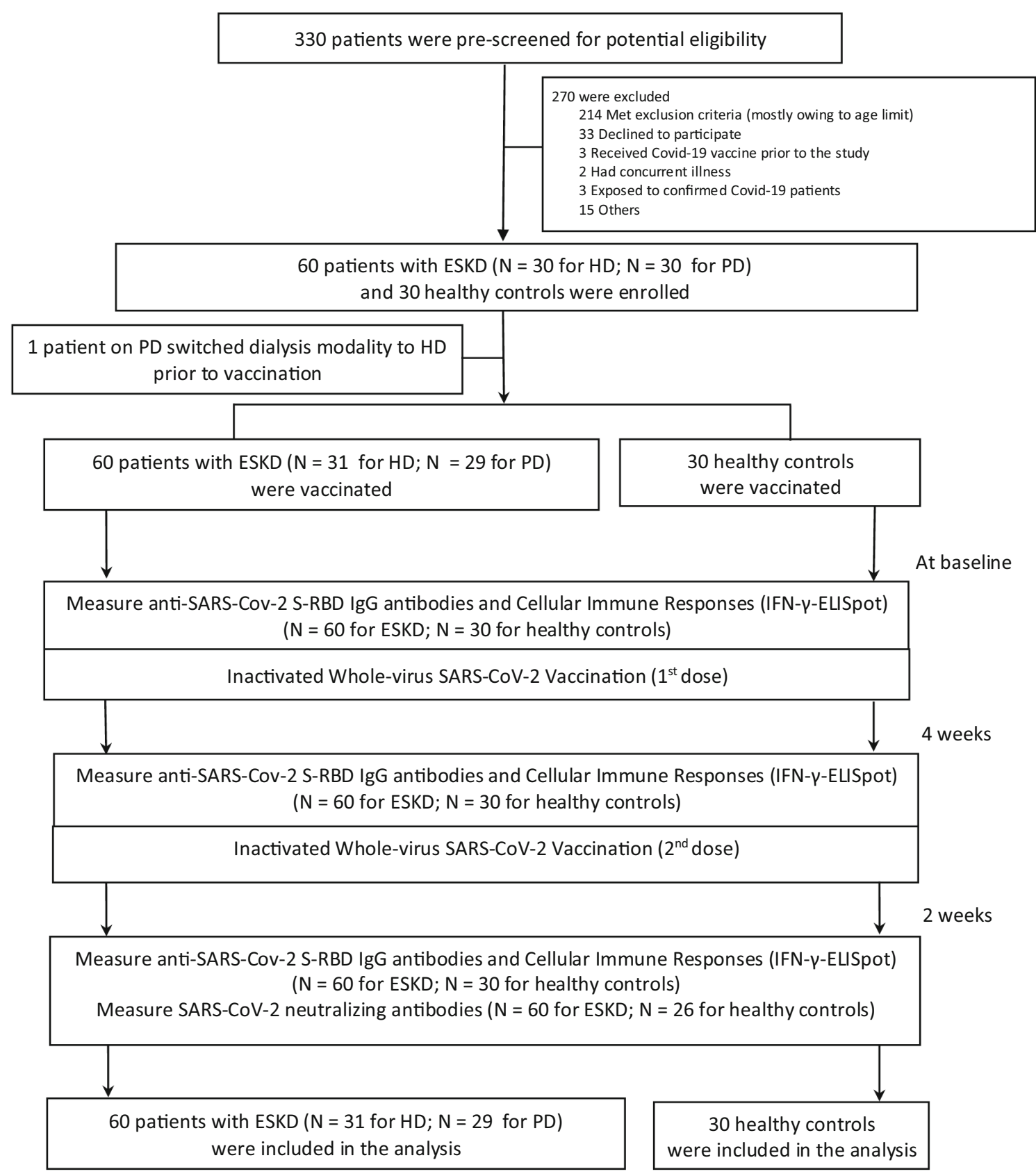

Fig. 1 Study design and flow diagram. The number of participants who were included in the study and analysis 
Table 1 Baseline characteristics of patients with ESKD undergoing HD and PD, and healthy controls

\begin{tabular}{|c|c|c|c|}
\hline Characteristics & HD $(n=31)$ & PD $(n=29)$ & $\begin{array}{l}\text { Healthy controls } \\
(n=30)\end{array}$ \\
\hline Age, years & $45(10)$ & $41(11)$ & $41(8)$ \\
\hline Male sex, $n(\%)$ & $23(74)$ & $17(59)$ & $23(77)$ \\
\hline Body mass index, $\mathrm{kg} / \mathrm{m}^{2}$ & $26(5)$ & $23(4)$ & $25(5)$ \\
\hline Weight, kg & $71(17)$ & $63(18)$ & $65(15)$ \\
\hline Age-adjusted Charlson Comorbidity Index, median (IQR) & $3(3-5)$ & $2(2-4)$ & $\mathrm{N} / \mathrm{A}$ \\
\hline Comorbidities, $n(\%)$ & & & $1(3)$ \\
\hline Diabetes mellitus & $14(45)$ & $7(24)$ & \\
\hline Hypertension & $24(77)$ & $25(86)$ & \\
\hline Coronary artery disease & $7(23)$ & $2(7)$ & \\
\hline Causes of ESKD, $n$ (\%) & & & N/A \\
\hline Diabetic nephropathy & $6(19)$ & $5(17)$ & \\
\hline Hypertensive nephropathy & $3(10)$ & $8(28)$ & \\
\hline Chronic glomerulonephritis & $5(16)$ & $8(28)$ & \\
\hline Others & $17(55)$ & $8(27)$ & \\
\hline Dialysis duration, months, median (IQR) & $33(17-84)$ & $32(7-55)$ & N/A \\
\hline Total $K t / V_{\text {urea }}$ & $1.6(0.4)$ & $2.0(0.4)$ & $\mathrm{N} / \mathrm{A}$ \\
\hline \multicolumn{4}{|l|}{ Residual kidney volume } \\
\hline Anuria, $n(\%)$ & $20(65)$ & $14(48)$ & N/A \\
\hline $\begin{array}{l}\text { Average residual urine volume in non-anuric patients } \\
(\mathrm{ml} / \text { day), median (IQR) }\end{array}$ & $600(400-1000)$ & $1000(600-1400)$ & \\
\hline Normalized protein catabolic rate, $\mathrm{g} / \mathrm{kg} / \mathrm{day}$ & $1.3(0.3)$ & $1.2(0.2)$ & N/A \\
\hline Smoking, $n(\%)$ & $14(45)$ & $10(35)$ & $2(7)$ \\
\hline \multicolumn{4}{|l|}{ Baseline laboratory data } \\
\hline White blood cells, $\times 10^{9} / 1$ & $6.9(1.8)$ & $7.3(2.7)$ & \\
\hline Absolute lymphocyte count, $\times 10^{9} / 1$ & $1.6(0.5)$ & $1.6(0.8)$ & \\
\hline Hemoglobin, $\mathrm{g} / \mathrm{dl}$ & $10.9(2.3)$ & $9.9(2.3)$ & \\
\hline Ferritin, ng/ml, median (IQR) & $301(119-441)$ & $351(172-734)$ & \\
\hline Albumin, g/l & $40.0(4.2)$ & $33.2(4.1)$ & \\
\hline Intact parathyroid hormone, $\mathrm{pg} / \mathrm{ml}$, median (IQR) & $393(212-812)$ & $441(115-763)$ & \\
\hline Calcium, mg/dl & $8.8(1.0)$ & $8.6(1.0)$ & \\
\hline Phosphorus, mg/dl & $5.5(1.9)$ & $5.4(1.9)$ & \\
\hline
\end{tabular}


Table 1 continued

\begin{tabular}{llll}
\hline Characteristics & HD $(\boldsymbol{n}=\mathbf{3 1})$ & PD $(\boldsymbol{n}=\mathbf{2 9 )}$ & $\begin{array}{l}\text { Healthy controls } \\
(\boldsymbol{n}=\mathbf{3 0})\end{array}$ \\
\hline C-reactive protein, mg/l, median (IQR) & $3.2(1.5-5.5)$ & $0.70(0.4-2.1)$ & \\
\hline
\end{tabular}

Values are mean (SD) unless otherwise indicated. Patients' characteristics between patients undergoing HD and PD were compared using the Student $t$ test and the Fisher's exact test for continuous and categorical variables, respectively. BMI was calculated from weight in kilograms divided by height squared. Anuria was defined as passing urine output of less than $100 \mathrm{ml}$ per day. Total $K t / V_{\text {urea }}$ represented total small-solute clearances

$E S K D$ end-stage kidney disease, $H D$ hemodialysis, $P D$ peritoneal dialysis, $I Q R$ interquartile range, $S D$ standard deviation

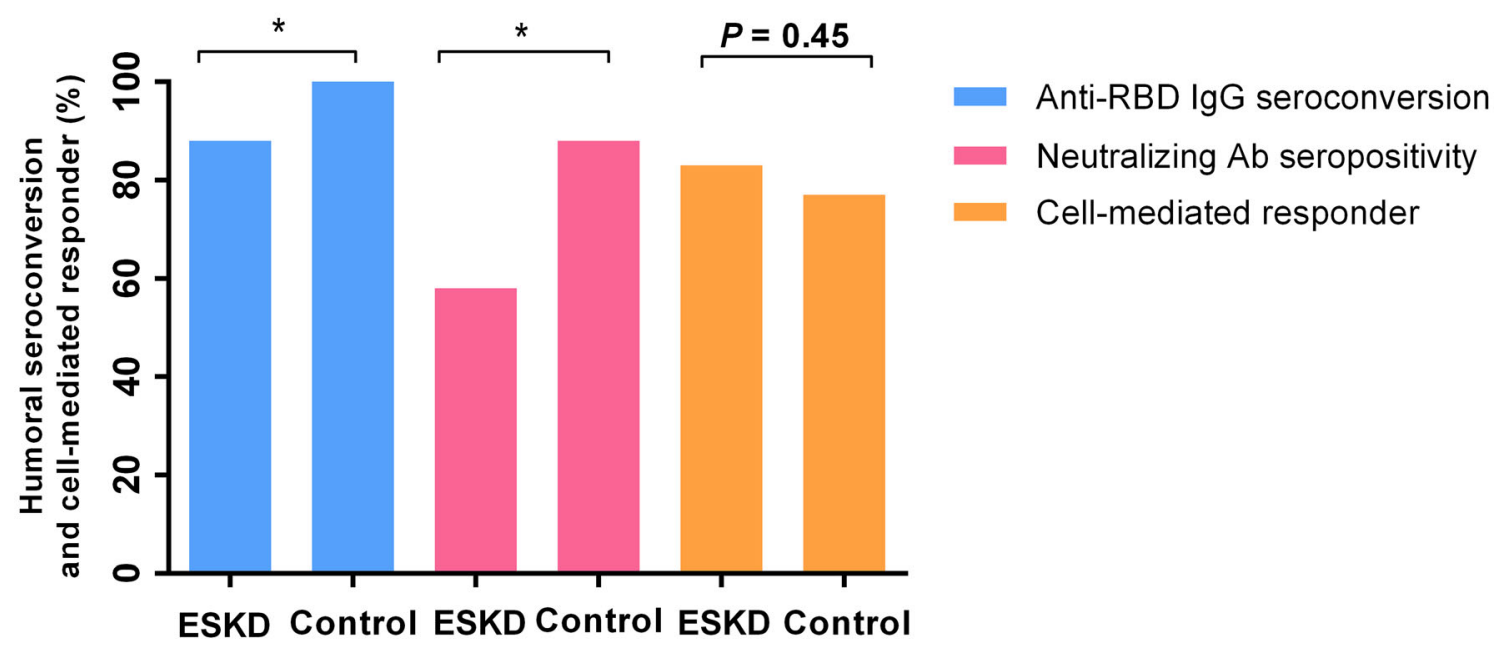

Fig. 2 Humoral seroconversion and cell-mediated immune responders among patients with ESKD and healthy controls after two vaccinations of inactivated whole-virus SARS-CoV-2 vaccine. The bar charts present

taken any form of immunosuppression, $5 \mathrm{mg}$ prednisolone daily. Baseline laboratory measurements were comparable between the two dialysis groups, apart from a lower mean $(\mathrm{SD})$ of serum albumin level in the PD group $(P<0.01)$. The mean (SD) age of healthy controls was 41 (8) years and $77 \%$ were men. None of the healthy controls were immunocompromised or receiving immunosuppressive therapies.

\section{SARS-CoV-2 Specific Humoral Immune Response}

After the second dose of vaccine, patients with ESKD achieved a lower seroconversion rate of percentages of patients who developed anti-RBD IgG seroconversion, had neuAb seropositivity, and were cellmediated immune responders at 2 weeks after the second dose of inactivated whole-virus SARS-CoV-2. ${ }^{*} P<0.05$

anti-RBD IgG compared to healthy controls ( $88 \%$ vs. $100 \%, P=0.05$ ) (Fig. 2 ). The change in anti-RBD IgG titers from baseline to 4 weeks after the first vaccination and 2 weeks after the second are shown in Fig. 3a. Similarly, the antiRBD IgG levels were significantly lower in patients with ESKD $(P<0.01)$. There were no significant differences in the anti-RBD IgG levels between patients undergoing $\mathrm{HD}$ and $\mathrm{PD}$ following the first $(P=0.35)$ or second dose $(P=0.11)$.

All participants in the ESKD group and 26 out of $30(87 \%)$ participants in the control group underwent assessment of neuAbs after completing the second vaccination. Patients 

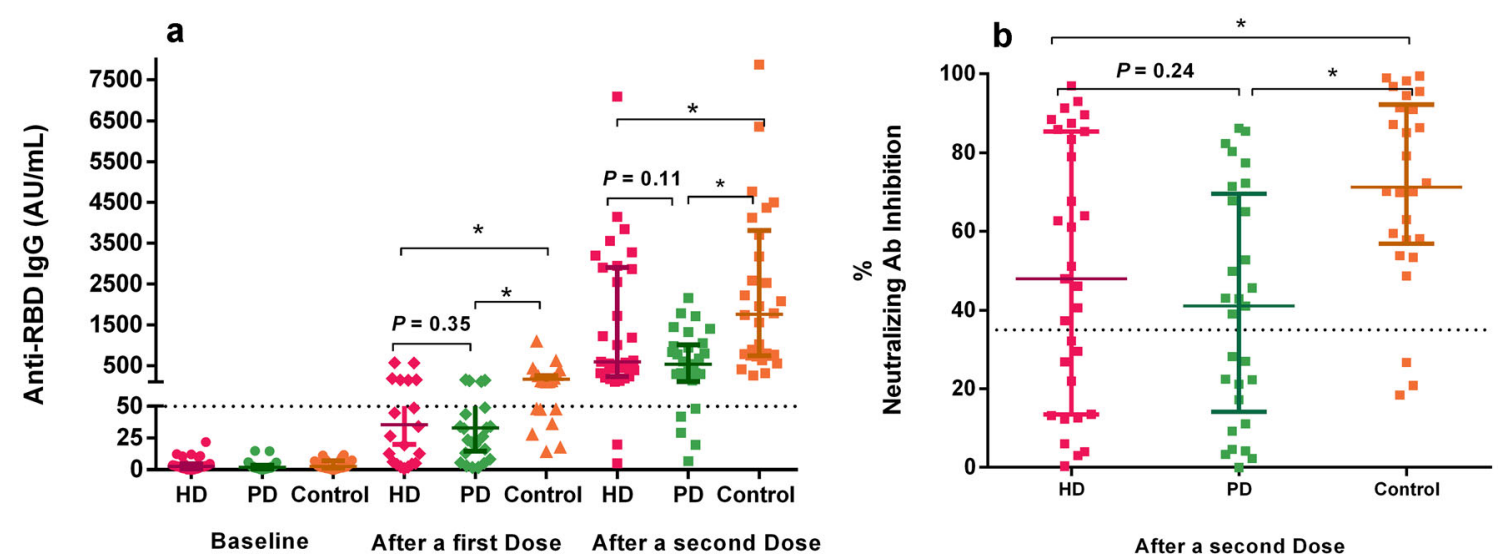

Fig. 3 a Anti-receptor-binding domain immunoglobulin $G$ titers and $\mathbf{b}$ percentages of surrogate virus neutralization $\mathrm{Ab}$ inhibition in patients with ESKD undergoing $\mathrm{HD}$ or $\mathrm{PD}$ and healthy controls. Anti-receptor-binding domain immunoglobulin $G$ titers were assessed pre-vaccination, 4 weeks after the first dose, and 2 weeks after the

with ESKD had a lower neuAb seropositivity rate compared with healthy controls $(58 \%$ vs. $88 \%$, $P=0.01$ ) (Fig. 2). The percentage of neuAb inhibition was also lower in patients with ESKD $(P<0.01)$ (Fig. 3b). Patients undergoing HD and PD had comparable levels of neuAb inhibition $(P=0.24)$ (Fig. 3b).

\section{Factors Associated with Neutralizing Ab Seronegativity Among Patients with ESKD}

Twenty-five (42\%) patients with ESKD had neuAb seronegativity after the second dose. Factors associated with being neuAb seronegative are summarized in Table 2. Age, absolute peripheral lymphocyte count, and serum ferritin level were associated with increased chances of being neuAb seronegative on univariate logistic analysis. In a multivariate model, all those factors remained significant. The odds of being neuAb seronegative were increased for older individuals (odds ratio [OR] 1.08; 95\% CI 1.02-1.16; $P=0.01$ ) and patients with higher serum ferritin level (OR 1.25 per every $100 \mathrm{ng} /$ $\mathrm{ml} ; 95 \%$ CI 1.02-1.53; $P=0.03$ ). Patients with ESKD who had absolute peripheral lymphocyte counts below $1.5 \times 10^{9}$ cells/l were five times second dose of inactivated whole-virus SARS-CoV-2 vaccine. Percentage neuAb inhibition was assessed 2 weeks after the second dose of inactivated whole-virus SARSCoV-2 vaccine. Bars represent medians with IQRs. ${ }^{*} P<0.05$

more likely to be neuAb seronegative (OR 5.17; 95\% CI 1.44-18.6; $P=0.01$ ).

\section{SARS-CoV-2 Specific Cell-Mediated Immune Response}

After the second vaccination, the proportions of cell-mediated immune responders against the S1 peptide pools in patients with ESKD were comparable to healthy controls (S1: 82\% vs. $77 \%, P=0.45$ ) (Fig. 2). Similarly, median S1-, S2N-, and SNMO-specific T cell responses after the second vaccination in patients with ESKD did not differ from controls $(P=0.13,0.89$, and 0.10 , respectively) (Fig. 4). Changes in SARSCoV-2-specific cell-mediated immune responses among patients undergoing $\mathrm{HD}, \mathrm{PD}$, and healthy controls following the first and the second dose of vaccine are shown in Fig. 4 . There were no differences in S1, S2N-, and SNMO-specific $\mathrm{T}$ cell responses between patients underoing HD and PD following the first or second dose.

\section{DISCUSSION}

Patients with ESKD are at high risk for severe COVID-19-related complications and mortality. 


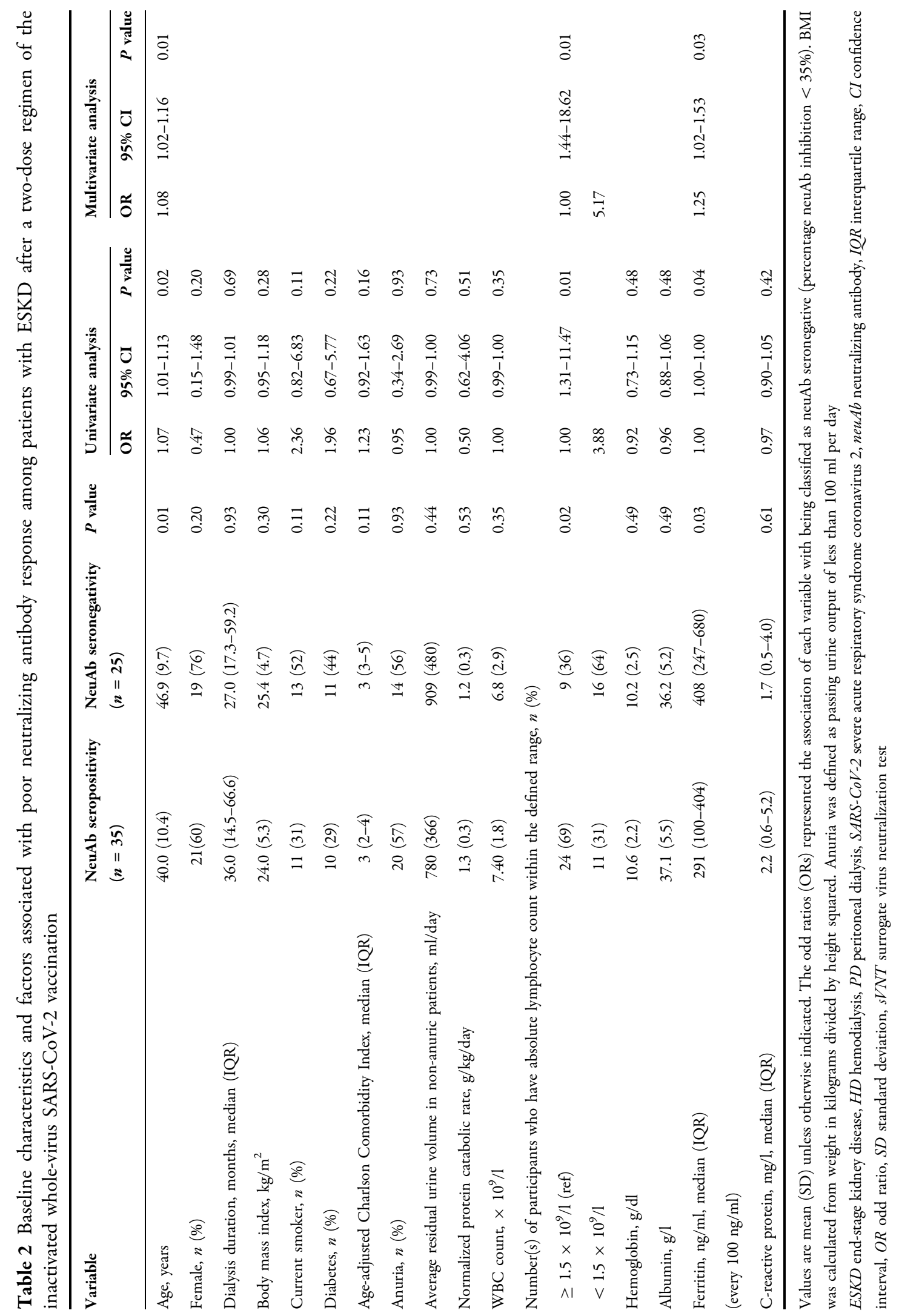




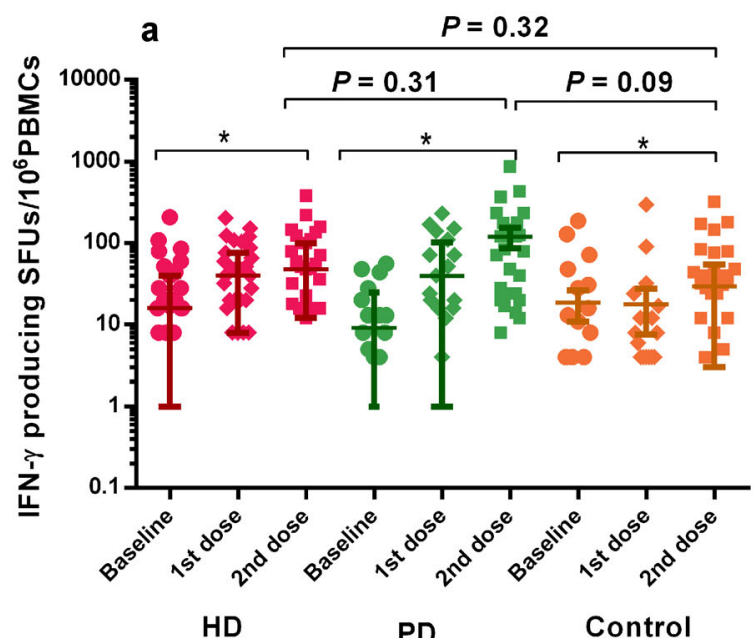

b

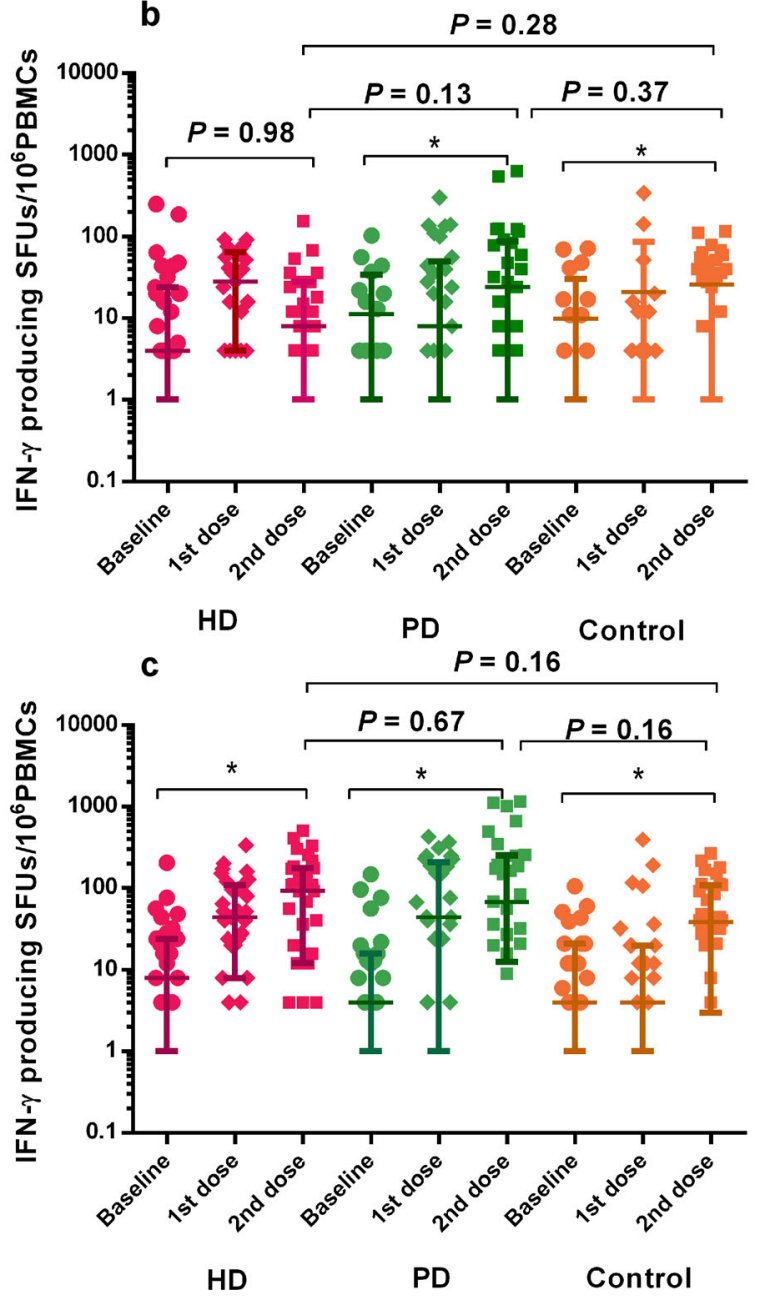

4 Fig. 4 The log-transformed SARS-CoV-2-specific T cell responses against a the $S 1$ protein, $\mathbf{b}$ the $S 2 \mathrm{~N}$ protein, and c the SNMO protein in patients with ESKD undergoing $\mathrm{HD}$ or PD and healthy controls at different time points. SARS-CoV-2-specific $\mathrm{T}$ cell responses detected by IFN $\gamma$ ELISpot were assessed before vaccination, 4 weeks after the first dose, and 2 weeks after the second dose of inactivated whole-virus SARS CoV-2 vaccine. Bars represent medians with IQRs. ${ }^{*} P<0.05$

Thus, vaccination against SARS-CoV-2 should be prioritized in this relatively immunocompromised population. In this study, we investigated SARS-CoV-2-specific humoral and cellmediated immune responses after two doses of the inactivated whole-virus SARS-CoV-2 vaccine, administered 1 month apart, in 60 patients undergoing dialysis. After two vaccinations, a satisfactory rate of anti-RBD IgG seroconversion but only a modest neuAb seropositivity were observed. In comparison to healthy controls, the rates of humoral seroconversion were lower and the magnitude of the humoral immune response was weaker in patients with ESKD. By contrast, percentages of cell-mediated immune responders and magnitude of the vaccine-elicited cellular immunity were similar among patients with ESKD and healthy controls.

An understanding of the humoral immune response to SARS-CoV-2 vaccines among patients with ESKD is rapidly evolving. Most studies use anti-RBD IgG titers or percentage viral neutralization as a surrogate measure of humoral immunity. A recent systematic review by Carr et al. [3] reported that the pooled estimate of antibody seropositivity following two doses of mRNA vaccines in patients with ESKD was $89 \%$ (95\% CI 85-91\%), a level comparable to the anti-RBD IgG seroconversion rate observed in our study. The high seroconversion rate of anti-RBD IgG observed in the patients with ESKD studied here may be explained by factors associated with the increased humoral immune response following COVID-19 vaccination $[5,7,8,19]$. Our patients with ESKD were slightly younger, with lower rates of immunosuppressive drug use compared to other series. 
Our data suggest that immunizing patients with ESKD who have factors favoring humoral immunity with an inactivated SARS-CoV-2 vaccine could achieve similar rates of anti-RBD IgG seroconversion achieved with mRNA-based vaccines. Nevertheless, in comparison to healthy controls, the attenuated humoral immune response to SARS-CoV-2 vaccination in patients with ESKD remained significantly lower [10]. Of note, all healthy controls in our cohort generated anti-RBD IgG seroconversion following a two-dose regimen of an inactivated SARS-CoV-2 vaccine. This finding is in line with an observational study that reports $100 \%$ SARS-CoV-2 IgG response in all healthy individuals following two doses of the BNT162b2 vaccine [20].

Although no acceptable neuAb threshold for protective immunity against SARS-CoV-2 is currently available, neuAb levels appear to correlate with subsequent immune protection against symptomatic SARS-CoV-2 infection [21]. The neuAb seropositivity rate in our cohort (58\% in the ESKD group and $88 \%$ in healthy controls) was much lower than the neuAb response achieved by the patients with ESKD who were vaccinated with a two-dose regimen of the BNT162b2 vaccine ( $82 \%$ among patients with ESKD and 100\% in healthy controls), using the same assay (sVNT) [20]. Approximately onethird of our patients with ESKD were still neuAb seronegative and likely unprotected after the standard two doses of the inactivated SARS$\mathrm{CoV}-2$ vaccine. Furthermore, they are potentially vulnerable to SARS-CoV-2 infection especially from new variants of COVID-19 viruses $[22,23]$. Among the various platforms of COVID-19 vaccines, the mRNA vaccines appear to induce the most robust neuAb response in patients with ESKD. Carr et al. [23] reported a stronger neuAb response in patients with ESKD who received the BNT162b2 vaccine compared to the AZD1222 vaccine. Furthermore, the proportion of patients with ESKD who generated neuAb response above the quantitative range (neuAb titer concentration needed to achieve 50\% inhibition, $\mathrm{IC}_{50}>40$ ) after two doses of the BNT162b2 vaccine and the ChAdOx1 nCoV-2 vaccine were higher than the neuAb seropositivity rate observed among patients with ESKD in our study. Hence, a higher dose of the inactivated SARS-CoV-2 vaccine or an additional heterologous booster vaccination may need to be considered in patients with ESKD following the standard two doses of the inactivated SARS-CoV-2 vaccine. Likewise, the neuAb seropositivity rate in our healthy controls (88\%) was lower compared to neuAb immune response observed in healthy volunteers following two doses of the BNT162b2 vaccine $(100 \%)$ and the ChAdOx1 nCoV-2 vaccine $(100 \%)$ [24, 25].

Factors associated with neuAb response in patients with ESKD are poorly understood. In this study, increasing age, high serum ferritin, and low absolute lymphocyte count were independently associated with a higher chance of being neuAb seronegative after being adjusted with other variables. We documented that predictors for being neuAb seronegative were similar to those of anti-RBD IgG. Increasing age and low peripheral lymphocyte count are associated with a lower rate of anti-RBD seroconversion $[7,8,19]$. Elevated serum ferritin may reflect chronic inflammatory state or iron storage status, factors that could affect the innate immune system [26]. However, serum albumin, dialysis vintage, and residual urine volume were not associated with neuAb response in our study.

Data on the cell-mediated immune response to SARS-CoV-2 vaccines among patients with ESKD is limited. The proportion of SARS-CoV-2 cell-mediated immune responders (82\%) against the $\mathrm{S} 1$ domain of spike protein observed in our study was lower compared to patients with ESKD who received a two-dose regimen of either the BNT162b2 or mRNA-1273 vaccines (100\% responders to SARS-CoV-2 spike protein S) [27]. The mRNA vaccines are designed to target the S1 domain of the spike protein. Therefore, patients receiving mRNA-based vaccines would be expected to mount greater S1specific $\mathrm{T}$ cell responses. In contrast, robust SNMO-specific T cell responses may be more potent following natural infection with SARSCoV-2 or after vaccination with inactivated SARS-CoV-2 vaccines. On the other hand, presenting the whole virus to the immune system might have the advantage of generating a broader immune response compared with other vaccine platforms that consist of only the spike protein, as with the ChAdOx1 nCoV-2 vaccine. 
However, the clinical benefit of presenting an inactivated whole virus SARS-CoV-2 vaccine over the mRNA or the adenovirus vector vaccines currently remains uncertain.

This is one of the first studies evaluating both humoral and cell-mediated immune responses of an inactivated whole-virus SARS-CoV-2 vaccine in patients requiring dialysis. For humoral immune response, we measured both anti-RBD IgG levels and percentage neuAb inhibition using an sVNT assay, a technique that correlates with the wild-type live-virus virus neutralization test [16]. Predictive factors for neuAb response were also examined. Antibody production is only one aspect of the protective immunity against viral infection. Although potential clinical utilities of evaluating cellular immune response after SARS-CoV-2 vaccination are poorly understood, durable and protective immune responses to subsequent viral infection require cell-mediated immunity. Hence, we attempted to assess cell-mediated immune responses to various SARS-CoV-2 proteins after vaccination which may mediate prolonged protective memory responses in these relatively immunocompromised patients, particularly when antibody levels wane over time. Our study had several limitations. First, we enrolled a relatively small number of participants which may increase the likelihood of a type II error. Second, our findings may not be generalizable to patients with ESKD outside of the age range of 18-59 years; these inclusion criteria were based on national Thailand regulatory requirements during the screening period. Third, comparisons of the immunogenicity among different studies are challenging because of varieties of techniques and criteria for anti-RBD IgG seroconversion and neuAb responsiveness. If antibody levels derived from different enzyme-linked immunosorbent or neutralization assays are referenced and expressed relative to the same international standard, direct comparisons of those parameters among studies can be achieved. Fourth, sVNT is more sensitive than the conventional virus neutralization test and sometimes detects non-neutralizing anti-RBD Abs. Consequently, titers of neuAb or rates of neuAb seropositivity obtained from studies using sVNT might be higher than those using conventional plaque-reduction neutralization tests $[16,28]$. Fifth, the immunogenicity profiles demonstrated in the present study represented only the initial immune response following vaccination with the inactivated whole-virus SARS-CoV-2 vaccine. Patients with ESKD receiving dialysis may have delayed immune responses after vaccination [29]. The short period between the second dose of vaccine to the time point of immunological response evaluation in this study could potentially affect the results. A longitudinal study of immune response after vaccination and vaccine effectiveness on clinical outcomes should be further explored.

\section{CONCLUSION}

Although two doses of the inactivated wholevirus SARS-CoV-2 vaccine can induce a comparable cellular immunity in patients with ESKD compared to healthy controls, the seroconversion rate and the magnitude of vaccine-elicited humoral immune response in patients with ESKD could remain suboptimal. Elderly patients with ESKD and high ferritinemia and lymphopenia are at increased risk of generating inadequate immune responses, and waning of the immunity would be expected sooner compared to those in better health. Therefore, further studies on SARS-CoV-2-specific immune monitoring after vaccination and a role of a booster dose of the SARS-CoV-2 vaccine are encouraged.

\section{ACKNOWLEDGEMENTS}

We thank Pongsan Chatsangcharoen and Parnwas Pinnobphun, MS, for laboratory technical support. We are also grateful to the infectious diseases nurses, dialysis nurses, and support staff at the Division of Infectious Diseases, Department of Medicine; the Clinical Research Center, Faculty of Medicine Ramathibodi Hospital, Mahidol University; and the Banphaeo General Hospital, Bangkok, Thailand. We also thank the participants of the study. 
Funding. This study was funded by the National Research Council of Thailand (NRCT), the Ministry of Higher Education, Science, Research, and Innovation of Thailand, and the Department of Medical Services, Ministry of Public Health of Thailand (102912). The Journal's Rapid Service Fee was funded by the authors.

Authorship. All named authors meet the International Committee of Medical Journal Editors (ICMJE) criteria for authorship for this article, take responsibility for the integrity of the work as a whole, and have given their approval for this version to be published.

Authors' Contributions. S. B., S. K, K. M., A. P., J. B. participated in conceptualization (create ideas and overarching research goal), S. B., A. N., M. A., J. B. participated in methodology. S. B., P. C., C. S., S. P., R. K. gathered data, S.B., A. N., M. A., J. B. analyzed data and drafted manuscript. A.D. carried out the supervision. All authors read and approved a final version of the manuscript.

Disclosures. Sarinya Boongird, Piyatida Chuengsaman, Chavachol Setthaudom, Arkom Nongnuch, Montira Assanatham, Salinnart Phanprasert, Rungthiwa Kitpermkiat, Sasisopin Kiertiburanakul, Kumthorn Malathum, Angsana Phuphuakrat, Andrew Davenport, and Jackrapong Bruminhent have nothing to disclose.

Compliance with Ethics Guidelines. The Institutional Review Board of the Faculty of Medicine, Ramathibodi Hospital, Mahidol University, Bangkok, Thailand approved the study protocol (approval number MURA2021/ 242). Participation in this study was discussed with the patients from Banphaeo General Hospital (Charoenkrung branch). The patients agreed to the referral, including sharing information about their condition and the Institutional Review Board of Banphaeo General Hospital (Charoenkrung branch) approved the release of patients' information. All participants provided written informed consent before enrollment. The study was conducted following the principles laid out in the declaration of Helsinki and was registered with the Thai Clinical Trials Registry (TCTR20210226002).

Data Availability. The datasets generated during and/or analyzed during the current study are available from the corresponding author on reasonable request.

Open Access. This article is licensed under a Creative Commons Attribution-NonCommercial 4.0 International License, which permits any non-commercial use, sharing, adaptation, distribution and reproduction in any medium or format, as long as you give appropriate credit to the original author(s) and the source, provide a link to the Creative Commons licence, and indicate if changes were made. The images or other third party material in this article are included in the article's Creative Commons licence, unless indicated otherwise in a credit line to the material. If material is not included in the article's Creative Commons licence and your intended use is not permitted by statutory regulation or exceeds the permitted use, you will need to obtain permission directly from the copyright holder. To view a copy of this licence, visit http:// creativecommons.org/licenses/by-nc/4.0/.

\section{REFERENCES}

1. Williamson EJ, Walker AJ, Bhaskaran K, et al. Factors associated with COVID-19-related death using OpenSAFELY. Nature. 2020;584(7821):430-6. https://doi.org/10.1038/s41586-020-2521-4.

2. Jager KJ, Kramer A, Chesnaye NC, et al. Results from the ERA-EDTA Registry indicate a high mortality due to COVID-19 in dialysis patients and kidney transplant recipients across Europe. Kidney Int. 2020;98(6):1540-8.

3. Carr EJ, Kronbichler A, Graham-Brown M, et al. Review of early immune response to SARS-CoV-2 vaccination among patients with CKD. Kidney Int Rep. 2021;6(9):2292-304. https://doi.org/10.1016/j. ekir.2021.06.027.

4. Yanay NB, Freiman S, Shapira M, et al. Experience with SARS-CoV-2 BNT162b2 mRNA vaccine in 
dialysis patients. Kidney Int. 2021;99(6):1496-8. https://doi.org/10.1016/j.kint.2021.04.006.

5. Stumpf J, Siepmann T, Lindner T, et al. Humoral and cellular immunity to SARS-CoV-2 vaccination in renal transplant versus dialysis patients: a prospective, multicenter observational study using mRNA-1273 or BNT162b2 mRNA vaccine. Lancet Reg Health Eur. 2021;9: 100178. https://doi.org/10. 1016/j.lanepe.2021.100178.

6. Rozen-Zvi B, Yahav D, Agur T, et al. Antibody response to SARS-CoV-2 mRNA vaccine among kidney transplant recipients: a prospective cohort study. Clin Microbiol Infect. 2021;27(8):1173.e11173.e4. https://doi.org/10.1016/j.cmi.2021.04. 028.

7. Longlune $N$, Nogier MB, Miedouge $M$, et al. High immunogenicity of a messenger RNA-based vaccine against SARS-CoV-2 in chronic dialysis patients. Nephrol Dial Transplant. 2021;36(9):1704-9. https://doi.org/10.1093/ndt/gfab193.

8. Grupper A, Sharon N, Finn $\mathrm{T}$, et al. Humoral response to the Pfizer BNT162b2 vaccine in patients undergoing maintenance hemodialysis. Clin J Am Soc Nephrol. 2021;16(7):1037. https://doi.org/10. 2215/CJN.03500321.

9. Agur T, Ben-Dor N, Goldman S, et al. Antibody response to mRNA SARS-CoV-2 vaccine among dialysis patients-a prospectivecohort study. Nephrol Dial Transplant. 2021. https://doi.org/10. 1093/ndt/gfab155.

10. Boongird S, Chuengsaman P, Phanprasert S, et al. Anti-SARS-CoV-2 spike protein S1 receptor-binding domain antibody after vaccination with inactivated whole-virus SARS-CoV-2 in end-stage kidney disease patients: an initial report. Kidney Int. 2021;100(5):1136-8. https://doi.org/10.1016/j.kint. 2021.08.007.

11. DaRoza G, Loewen A, Djurdjev O, et al. Stage of chronic kidney disease predicts seroconversion after hepatitis B immunization: earlier is better. Am J Kidney Dis. 2003;42(6):1184-92. https://doi.org/10. 1053/j.ajkd.2003.08.019.

12. Eiselt J, Kielberger L, Rajdl D, Racek J, Pazdiora P, Malánová L. Previous vaccination and age are more important predictors of immune response to influenza vaccine than inflammation and iron status in dialysis patients. Kidney Blood Press Res. 2016;41(2):139-47. https://doi.org/10.1159/ 000443416 .

13. Nongnuch A, Ngampongpan W, Srichatrapimuk S, et al. Immune response to influenza vaccination in ESRD patients undergoing hemodialysis vs. hemodiafiltration. PLOS ONE. 2020;15(2): e0227719. https://doi.org/10.1371/journal.pone. 0227719 .

14. Abbott Core Laboratory. Abbott Architect i SARSCoV-2 IgG II Quant Reagent Instructions for Use. 2012. https://www.corelaboratory.abbott/int/en/ offerings/segments/infectious-disease/sars-cov-2. Accessed 30 Sep 2021.

15. Chew KL, Tan SS, Saw S, et al. Clinical evaluation of serological IgG antibody response on the Abbott Architect for established SARS-CoV-2 infection. Clin Microbiol Infect. 2020;26(9):1256.e9-1256. e11. https://doi.org/10.1016/j.cmi.2020.05.036.

16. Tan CW, Chia WN, Qin X, et al. A SARS-CoV-2 surrogate virus neutralization test based on antibody-mediated blockage of ACE2-spike protein-protein interaction. Nat Biotechnol. 2020;38(9):1073-8. https://doi.org/10.1038/ s41587-020-0631-z.

17. Coronavirus diagnostics by Euroimmun. SARSCoV-2 NeutraLISA. https://www.coronavirusdiagnostics.com/documents/Indications/ Infections/Coronavirus/EI_2606_D_UK_F.pdf. Accessed 7 Sep 2021.

18. Zuo J, Dowell AC, Pearce H, et al. Robust SARS-CoV2-specific $\mathrm{T}$ cell immunity is maintained at 6 months following primary infection. Nat Immunol. 2021;22(5):620-6. https://doi.org/10.1038/s41590021-00902-8.

19. Broseta JJ, Rodríguez-Espinosa D, Rodríguez N, et al. Humoral and cellular responses to mRNA-1273 and BNT162b2 SARS-CoV-2 vaccines administered to hemodialysis patients. Am J Kidney Dis. 2021;78(4): 571-81. https://doi.org/10.1053/j.ajkd.2021.06. 002.

20. Speer C, Goth D, Benning L, et al. Early humoral responses of hemodialysis patients after COVID-19 vaccination with BNT162b2. Clin J Am Soc Nephrol. 2021;16(7):1073-82. https://doi.org/10. 2215/CJN.03700321.

21. Khoury DS, Cromer D, Reynaldi A, et al. Neutralizing antibody levels are highly predictive of immune protection from symptomatic SARS-CoV-2 infection. Nat Med. 2021;27(7):1205-11. https://doi.org/ 10.1038/s41591-021-01377-8.

22. Planas D, Veyer D, Baidaliuk A, et al. Reduced sensitivity of SARS-CoV-2 variant delta to antibody neutralization. Nature. 2021;596(7871):276-80. https://doi.org/10.1038/s41586-021-03777-9.

23. Carr EJ, Wu M, Harvey R, et al. Neutralising antibodies after COVID-19 vaccination in UK haemodialysis patients. Lancet. 2021;398(10305): 
$1038-41$. 6736(21)01854-7.

https://doi.org/10.1016/S0140-

24. Wall EC, Wu M, Harvey R, et al. Neutralising antibody activity against SARS-CoV-2 VOCs B.1.617.2 and B.1.351 by BNT162b2 vaccination. Lancet. 2021;397(10292):2331-3. https://doi.org/10.1016/ s0140-6736(21)01290-3.

25. Wall EC, Wu M, Harvey R, et al. AZD1222-induced neutralising antibody activity against SARS-CoV-2 Delta VOC. Lancet. 2021;398(10296):207-9. https://doi.org/10.1016/s0140-6736(21)01462-8.

26. James EC, Eric PS. Iron in infection and immunity. Cell Host Microbe. 2013;13(5):509-19. https://doi. org/10.1016/j.chom.2013.04.010.

27. Bertrand D, Hamzaoui M, Lemée V, et al. Antibody and $\mathrm{T}$ cell response to SARS-CoV-2 messenger RNA
BNT162b2 vaccine in kidney transplant recipients and hemodialysis patients. J Am Soc Nephrol. 2021;32(9):2147. https://doi.org/10.1681/ASN. 2021040480 .

28. Valcourt EJ, Manguiat K, Robinson A, et al. Evaluation of a commercially-available surrogate virus neutralization test for severe acute respiratory syndrome coronavirus-2 (SARS-CoV-2). Diagn Microbiol Infect Dis. 2021;99(4): 115294. https://doi.org/ 10.1016/j.diagmicrobio.2020.115294.

29. Schrezenmeier E, Bergfeld L, Hillus D, et al. Immunogenicity of COVID-19 Tozinameran vaccination in patients on chronic dialysis. Front Immunol. 2021;12: 690698. https://doi.org/10. 3389/fimmu.2021.690698. 